\title{
Cardiometabolic Factors and Breast Cancer: A Case-Control Study in Women
}

\author{
Niki Mourouti ${ }^{1}$, Christos Papavagelis ${ }^{1}$, Meropi D. Kontogianni ${ }^{1}$, Petrini Plytzanopoulou ${ }^{2}$, \\ Tonia Vassilakou ${ }^{2}$, Nikolaos Malamos ${ }^{3}$, Athena Linos ${ }^{4}$ and Demosthenes B. Panagiotakos ${ }^{1, *}$
}

\author{
${ }^{I}$ Department of Nutrition and Dietetics, Harokopio University, Athens, Greece ${ }^{2}$ Department of Nutrition and Biochemis- \\ try, National School of Public Health, Athens, Greece; ${ }^{3}$ Pathology-Oncology Department, General Hospital "Elena \\ Venizelos", Athens, Greece; ${ }^{4}$ Department of Hygiene, Epidemiology and Medical Statistics, School of Medicine, Univer- \\ sity of Athens, Athens, Greece
}

\begin{abstract}
Background: Previous studies have suggested that individual cardiometabolic factors may be associated with an increased risk of breast cancer. Objective: To evaluate the association between individual cardiometabolic factors with breast cancer development. Design: A case - control study. Two-hundred-and-fifty consecutive, newly diagnosed breast cancer female patients ( $56 \pm 12$ years) and 250 , one-to-one age-matched with the patients, healthy volunteers (controls), were studied. A standardized, validated questionnaire assessing various socio-demographic, clinical, lifestyle and dietary characteristics, was applied through face-to-face interviews. Adherence to the Mediterranean diet was evaluated using the 11-components MedDietScore (theoretical range 0-55). A detailed medical history regarding the common co-morbidities (i.e., diabetes, hypertension, hypercholesterolemia) and their treatment was also recorded, while women were also categorized using the Body Mass Index (BMI) as an indicator of obesity. Results: Obesity (i.e., BMI $>30 \mathrm{~kg} / \mathrm{m}^{2}$ ) was positively associated with the likelihood of having breast cancer. Conclusions: With the exception of obesity, none of the other tested cardiometabolic risk factors seemed to be a predisposing factor for breast cancer development.
\end{abstract}

Keywords: Cardiometabolic factors, diabetes, hypercholesterolemia, hypertension, obesity.

\section{INTRODUCTION}

Metabolic syndrome is defined (by the American Heart Association-AHA and the National Heart, Lung and Blood Institute-NHLBI) as the clustering of three or more of the following conditions: abdominal obesity (waist circumference $\geq 88 \mathrm{~cm}$ ), elevated blood pressure $(\geq 130 \mathrm{mmHg}$ systolic over $\geq 85 \mathrm{mmHg}$ diastolic), elevated fasting glucose $(\geq 100 \mathrm{mg} / \mathrm{dL})$, hypertriglyceridemia ( $\geq 150 \mathrm{mg} / \mathrm{dL})$, and low high-density lipoprotein (HDL) cholesterol $(<50 \mathrm{mg} / \mathrm{dL})$ [1]. During the last 30 years, metabolic syndrome has been widely associated with human health. Specifically, several studies have suggested that the metabolic syndrome is an important risk factor for cardiovascular disease incidence and mortality, as well as all-cause mortality $[2,3]$. In a very recent systematic review and meta-analysis, the metabolic syndrome was associated with a 2 -fold increase in cardiovascular outcomes and a 1.5 -fold increase in all-cause mortality [4]. Moreover, individual cardiometabolic factors such as obesity [5-7], diabetes [8,9] and high blood pressure [10] have been also associated with increased risk of cardiovascular disease.

Cancer is a chronic disease that together with cardiovascular disease is responsible for the vast majority of deaths in the developed world, and recently in developing countries, as

*Address correspondence to this author at the 46 Paleon Polemiston St., 16674, Glyfada, Greece; Tel: +30 210 9603116; Fax: +30 210 9600719; E-mail: d.b.panagiotakos@usa.net well. Among various types of cancers, breast cancer is the most frequently diagnosed cancer and the leading cause of cancer deaths in females, worldwide. However, its relationship with the metabolic syndrome and even more specifically with individual cardiometabolic factors remains not well understood and appreciated. In a recent systematic review and meta-analysis that aimed to assess the association between metabolic syndrome and risk of cancer at different sites, the presence of metabolic syndrome was positively associated with breast cancer postmenopausally [11]. Moreover, in a longitudinal exploring the association between the metabolic syndrome and the risk of postmenopausal breast cancer, the association was assessed among women in the $6 \%$ sample of subjects in the Women's Health Initiative clinical trial and the $1 \%$ sample of women in the observational study who had repeated measurements of the components of the syndrome during follow-up. In timedependent covariate an analysis, a positive association between the metabolic syndrome and breast cancer was indicated, due primarily to positive associations with serum glucose, serum triglycerides and diastolic blood pressure [12]. In contrast, in the MEtabolic syndrome and CANcer (MeCan) project, a large prospective study conducted in women from Austria, Norway and Sweden, analyses of risk of breast cancer incidence showed that there was a decreased risk of incident breast cancer in women below age 50 with high Body Mass Index (BMI). Furthermore, there was an inverse association of most Metabolic Syndrome components with breast cancer before age 50, and only glucose was positively associated with risk [13]. 
Thus, the aim of this work was to evaluate the association between individual cardiometabolic factors (i.e., components of the Metabolic Syndrome) with breast cancer development after various adjustments made.

\section{MATERIALS AND METHODS}

\section{Study's Design and Sample}

This is a case-control study, with face-to-face interviews with the participants. Between November $1^{\text {st }}, 2010$ and July $31^{\text {st }}, 2012,250$ consecutive, newly (within six months) diagnosed breast cancer female patients (defined by physical examination and biopsy) that visited pathology-oncology clinics of five major General Hospitals in Athens, Greece (i.e., "Alexandra" General Hospital, "Elena Venizelos" Maternity-General Hospital, "Agioi Anargyroi" General Oncological Hospital of Kifissia, "Saint Savvas" Cancer Hospital and "I. Metaxa" Special Cancer Hospital) were contacted to participate in the study. Patients with diagnosis older than six months (in order to avoid changes in their dietary habits or other behaviours), were not included. In the same period, 250 female subjects (controls) without any clinical symptoms, signs or suspicious of any type of cancer in their medical history, were selected on a volunteer basis. The control subjects were allocated at population basis from their work or home places and were age-matched ( \pm 3 years) with the cancer patients.

The number of the enrolled subjects $(n=500)$ was decided through power analysis, in order to evaluate (two sided) odds ratio equal to $1.10(95 \% \mathrm{CI} 1.05,1.15)$, achieving statistical power greater than 0.80 at 0.05 probability level ( $p$-value).

The design of the study and the analytical methods followed have been already described elsewhere [14].

\section{Bioethics}

The study has been approved by the Ethics Committee of "Alexandra" General Hospital (No. 4/10.3.2010), "I. Metaxa" Special Cancer Hospital (No. 40/8.12.2011) and "Saint Savvas" Cancer Hospital (No. 448/2.3.2012) and was carried out in accordance to the Declaration of Helsinki (1989) of the World Medical Association. Prior to the collection of any information, participants were informed about the aims and procedures of the study and provided their signed consent.

\section{Dietary Assessment}

A validated, semi-quantitative food frequency questionnaire (FFQ) was used during the interviews to collect dietary information from the participants [14]. In brief, the FFQ included 86 questions regarding the frequency of consumption of all main food groups and beverages usually consumed. Specifically, data on regular consumption of each food group (i.e., never, rarely, 3-4 times/month, 1-2 times/week, 3-4 times/week, daily), were recorded for the last year prior to diagnosis. Adherence to the Mediterranean dietary pattern was assessed using the MedDietScore, an 11-item composite dietary index, with large scale scoring that ensures better predictive accuracy [15]. The range of the MedDietScore is between 0 and 55. Higher values of this score indicate greater adherence to the Mediterranean diet. The validation properties of the MedDietScore have been presented elsewhere in the literature [15-17].

\section{Other Measurements}

Age of the participants was recorded, as well as their educational level and financial status. Physical activity was assessed using the International Physical Activity Questionnaire (IPAQ) index [18] that has been validated for the Greek population [19]. Subjects were asked to recall the number of days and hours or minutes they engaged in physical activity of different intensities for at least ten minutes, vigorous intensity and moderate intensity, walking and time spent sitting. According to their physical activity levels, participants were classified as inactive, minimally active or health enhancing physical activity (HEPA) active. Smoking habits (i.e. current and former smoking, total years of smoking and number of cigarettes smoked per day) were also recorded. A previously translated and validated version of the Zung Depression Rating Scale (ZDRS) was used for the assessment of depressive symptoms [20,21], while anxiety was assessed with the also previously translated and validated version of the Spielberger Trait Anxiety Inventory (STAI from Y-2), which is a 20 -item self-reported questionnaire evaluating how the respondent feels generally $[22,23]$. Family history of breast cancer as well as gynaecological medical history (i.e., existence or not of menstruation, age of menarche, age of menopause and use of hormone replacement therapy), were recorded during the interview.

\section{Cardiometabolic Factors}

Weight and height were measured and body mass index was calculated. Afterwards, women were categorized as normal weight $\left(18.5 \mathrm{~kg} / \mathrm{m}^{2}<\mathrm{BMI}<24.9 \mathrm{~kg} / \mathrm{m}^{2}\right)$, overweight (25 $\mathrm{kg} / \mathrm{m}^{2}<$ BMI $<29.9 \mathrm{~kg} / \mathrm{m}^{2}$ ), and obese (BMI $>30$ $\left.\mathrm{kg} / \mathrm{m}^{2}\right)$. Furthermore, a detailed medical history regarding the common co-morbidities and their treatment was also recorded during the interview. More specifically, participants were asked if they had been diagnosed with any of a list of medical conditions that included diabetes, hypertension and hypercholesterolemia. They were also asked to report medications used every week to treat the above medical conditions, such as insulin, pills to treat diabetes, diuretics, blood pressure lowering drugs as well as cholesterol-lowering medications.

\section{Statistical Analysis}

Continuous variables that were normally distributed are presented as mean \pm SD. Normality was evaluated using the P-P plots. Skewed variables are presented as medians and quartiles and categorical variables as frequencies. Associations between categorical variables were tested by the calculation of chi-squared test. Student's t-test for independent samples was used to evaluate mean differences between normally distributed variables (i.e., MedDietScore), where in case of skewed continuous variables (i.e., IPAQ score), the tested hypothesis was evaluated using the non-parametric Utest suggested by Mann and Whitney. Multiple logistic regression analysis was applied to evaluate the association of the adherence to the Mediterranean diet in relation to the likelihood of having breast cancer. The results are presented 
as odds ratios (OR) and their $95 \%$ corresponding confidence intervals $(95 \% \mathrm{CI})$. All reported p-values were based on twosided tests. Statistical calculations were performed with SPSS 18 software (SPSS Inc., Chicago, IL, USA).

\section{RESULTS}

\section{Characteristics of Patients and Controls}

In Table (1) the basic characteristics of patients and controls are presented. Cases and controls were of similar age (according to the protocol of the study), while educational and financial status were lower in cases as compared to the controls $(\mathrm{p}<0.001)$. The level of adherence to the Mediterranean diet was moderate in both cases (i.e., 28/55) and controls (i.e., 30/55). On the other hand, cases were more likely to have at least one relative with breast cancer. There was no significant difference in smoking habits $(\mathrm{p}=0.207)$, as well as in menopausal status $(\mathrm{p}=0.49)$, while cases reported lower physical activity status (i.e., lower IPAQ score).

Regarding the main research hypothesis, no significant association was observed between diabetes, hypertension, hypercholesterolemia and presence of breast cancer $(p>0.05)$, while obesity was marginally associated with the disease. In particular, cases were more likely to be obese (BMI> $\left.30 \mathrm{~kg} / \mathrm{m}^{2}\right)$ as compared to controls $(\mathrm{p}=0.057)$ (Table 2). Unadjusted logistic regression analysis revealed that compared to normal weight, obesity (i.e. BMI $>30$ $\mathrm{kg} / \mathrm{m}^{2}$ ), but not overweight was associated with higher likelihood of having breast cancer (odds ratio $=1.641, \mathrm{p}=0.041$, odds ratio $=0.988, \mathrm{p}=0.954$, respectively).

\section{Cardiometabolic Factors and Breast Cancer}

Three additive, multiple logistic regression models were fitted, in order to evaluate the association between the basic cardiometabolic factors and the likelihood of having breast cancer (Table 3). The use of additive models assisted in better exploring the potential moderating effect of various factors in the investigated relationships. The first model included as potential confounders only age, obesity, diabetes, hypertension and hypercholesterolemia. It was observed that obesity (i.e. BMI $>30 \mathrm{~kg} / \mathrm{m}^{2}$ ) was positively associated with the likelihood of having breast cancer (Odds Ratio $=1.71$, $\mathrm{p}=0.033$ ). In the second model, smoking ever (yes/no), physical activity (in MET-minutes/week), family history of breast cancer in order to encompass the genetic predisposition and potential lifestyle changes (yes/no), as well as menopausal status (pre vs. postmenopausal) that has been strongly associated with breast cancer incidence, were also entered. No significant association was observed between obesity, diabetes, hypertension, hypercholesterolemia and the likelihood of having breast cancer $(p>0.05)$. In the third model, the MedDietScore (that evaluates adherence to a healthy dietary pattern, the Mediterranean), was further included. The lack of association between the basic cardiometabolic factors and the likelihood of having breast cancer continued to exist $(\mathrm{p}>0.05)$.

\section{Profile Analysis of the Association of the Cardiome- tabolic Factors and the Likelihood of Breast Cancer}

Multi-adjusting cannot entirely exclude residual confounding. Thus, sub-group analysis by menopausal status

Table 1. Distribution of Patients' and Controls' Characteristics

\begin{tabular}{|c|c|c|c|}
\hline & Breast cancer cases & Controls & $p$ \\
\hline $\mathbf{N}$ & 250 & 250 & \\
\hline Age (years) (mean \pm standard deviation) & $56 \pm 12$ & $56 \pm 12$ & 0.99 \\
\hline Years of education (years) (mean \pm standard deviation) & $11 \pm 4$ & $12 \pm 4$ & $<0.001$ \\
\hline Financial status $(0-10)($ mean \pm standard deviation $)$ & $5 \pm 2$ & $6 \pm 2$ & $<0.001$ \\
\hline MedDietScore (0-55) (mean \pm standard deviation) & $27.8 \pm 5$ & $29.9 \pm 4$ & $<0.001$ \\
\hline Smoking (ever), (n. \%) & $103(41.2 \%)$ & $117(46.8 \%)$ & 0.207 \\
\hline Number of relatives with breast cancer, (n. \%) & & & 0.003 \\
\hline No relative & $177(71.1 \%)$ & $211(84.7 \%)$ & \\
\hline 1 relative & $62(24.9 \%)$ & $32(12.9 \%)$ & \\
\hline 2 relatives & $9(3.6 \%)$ & $5(2.0 \%)$ & \\
\hline 3 relatives & $0(0 \%)$ & $1(0.4 \%)$ & \\
\hline 5 relatives & $1(0.4 \%)$ & $0(0 \%)$ & \\
\hline Menopausal status (n. \%) & & & 0.49 \\
\hline Premenopausal women & $84(33.6 \%)$ & $91(36.5 \%)$ & \\
\hline Postmenopausal women & $166(66.4 \%)$ & $158(63.5 \%)$ & \\
\hline IPAQ score (MET-minutes/week) & $219(0.00,985.5)$ & $876(140.25,1533.0)$ & $<0.001$ \\
\hline
\end{tabular}

The MedDietScore is a measurement of adherence to the Mediterranean diet, with theoretical range 0-55; greater values reporting greater adherence to this pattern.

The reported $p$-values were calculated using the t-test, the chi-square test or the Mann-Whitney U test. 
Table 2. Distribution of Patients' and Controls' Cardiometabolic Factors

\begin{tabular}{|c|c|c|c|}
\hline & Breast Cancer Cases & Controls & $p$ \\
\hline Normal weight women $\left(18.5 \mathrm{~kg} / \mathrm{m}^{2}<\mathrm{BMI}<24.9 \mathrm{~kg} / \mathrm{m}^{2}\right)$ & $79(33.8 \%)$ & $93(38 \%)$ & \\
\hline Overweight women $\left(25 \mathrm{~kg} / \mathrm{m}^{2}<\mathrm{BMI}<29.9 \mathrm{~kg} / \mathrm{m}^{2}\right)$ & $84(35.9 \%)$ & $101(41.2 \%)$ & \\
\hline Diabetes (n. \%) & $12(4.8 \%)$ & $8(3.2 \%)$ & 0.361 \\
\hline Hypertension (n. \%) & $33(13.2 \%)$ & $34(13.6 \%)$ & 0.896 \\
\hline Hypercholesterolemia (n. \%) & $18(7.2 \%)$ & $17(6.8 \%)$ & 0.861 \\
\hline
\end{tabular}

The reported $p$-values were calculated using the chi-square test.

Table 3. Results from Multiple Logistic Regression Models that were Applied to Evaluate the Association of Cardiometabolic Factors with the Likelihood of having Breast Cancer in Cases $(n=250)$ and Controls $(n=250)$. Results are Presented as Odds Ratios and $95 \% \mathrm{CI}$

\begin{tabular}{|c|c|c|c|}
\hline & Model 1 & Model 2 & Model 3 \\
\hline Normal weight women & 1.00 & 1.00 & 1.00 \\
\hline Overweight women & $1.02(0.66,1.57)$ & $0.93(0.60,1.46)$ & $0.92(0.59,1.46)$ \\
\hline Diabetes (yes vs. no) & $1.33(0.50,3.50)$ & $1.25(0.47,3.35)$ & $1.36(0.50,3.73)$ \\
\hline Hypertension (yes vs. no) & $0.93(0.52,1.65)$ & $0.90(0.49,1.63)$ & $0.87(0.47,1.61)$ \\
\hline Hypercholesterolemia (yes vs. no) & $1.02(0.49,2.11)$ & $1.01(0.47,2.14)$ & $0.92(0.43,1.97)$ \\
\hline Menopausal status (pre vs. postmenopausal) & - & $1.22(0.69,2.16)$ & $1.19(0.67,2.13)$ \\
\hline MedDietScore (per 1/55 units) & - & - & $0.91(0.88,0.95)$ \\
\hline
\end{tabular}

All odds ratios and their corresponding $95 \%$ confidence intervals were calculated by performing multiple logistic regressions.

was applied. The results were similar to the ones presented above; particularly, both for premenopausal and postmenopausal women, neither of the individual cardiometabolic factors (i.e., obesity, diabetes, hypertension and hypercholesterolemia) was associated with the likelihood of having breast cancer $(p>0.05)$. When the analysis was stratified according to the existence of each one o the individual cardiometabolic factors (i.e., diabetes, hypertension, hypercholesterolemia) in order to observe the mediating effect of obesity, the results were similar. In both diabetic and nondiabetic women, BMI was not significantly associated with the likelihood of having breast cancer (all p's $>0.05$ ). Moreover, when the analysis was stratified according to the exis- tence of hypertension, BMI was marginally associated with an increased likelihood of having breast cancer $(\mathrm{OR}=1.046$, $\mathrm{p}=0.05$ ) for those women with no history of hypertension, while for those who had a history of hypertension, there was no significant association $(\mathrm{p}=0.05)$. At last, stratified analysis by history of hypercholesterolemia revealed no significant association of BMI with breast cancer $(\mathrm{p}>0.05)$.

\section{DISCUSSION}

Based on the presented findings, only obesity was associated with increased likelihood of having breast cancer. The detrimental effect of excess body weight, as measured by 
various means, i.e., BMI, percentage of body fat, waist circumference or waist-hip ratio, on breast cancer development has been reported by some other observational studies, too. In a recent meta-analysis conducted to estimate the overall effect of overweight and obesity on breast cancer during pre- and postmenopausal period, a direct and significant correlation during postmenopausal period was revealed (Odds Ratio $=1.15 ; 95 \%$ Confidence Interval $(\mathrm{CI})$ 1.07, 1.24; Risk Ratio (RR) = 1.16; 95\% CI 1.08, 1.25; and Rate Ratio $=0.98 ; 95 \%$ CI $0.88,1.09)$ [24]. Moreover, in a cohort study with 7.723 women conducted to determine whether higher adiposity is associated with greater breast cancer risk in older postmenopausal women, women in the uppermost quartiles of weight, weight gain since age 25, body mass index, waist circumference, and percentage of body fat had higher breast cancer rates than women in the first quartiles of each measure. Specifically, breast cancer rates were $49 \%$ higher for women in the uppermost quartile of weight (hazard ratio $(\mathrm{HR})=1.49,95 \% \mathrm{CI}=1.05-2.10$ ) and $58 \%$ higher for women in the top quartile of percentage of body fat $(\mathrm{HR}=1.58,95 \% \mathrm{CI}=1.11-2.23)$ than for women in the lowest quartile of each measure. The associations between adiposity measures and breast cancer rates were not altered when the analyses were limited to very elderly women $(>$ or $=70)$ [25]. Furthermore, Lahmann et al., [26] who used data from 73.542 premenopausal and 103.344 postmenopausal women from 9 European countries taking part in the EPIC (European Prospective Investigation into Cancer and Nutrition) study, concluded that in postmenopausal women not taking exogenous hormones, weight, BMI and hip circumference were positively associated with breast cancer risk. Similarly, in the Women's Health Initiative Observational Study (WHI-United States) researchers found that generalized obesity is an important risk factor for postmenopausal breast cancer (Relative risk $(\mathrm{RR})=2.52 ; 95 \% \mathrm{CI}=1.62-3.93$ for heavier women with baseline $\mathrm{BMI}>31.1$ compared to slimmer women with baseline BMI <22.6) [27]. However, in a very recent systematic review and meta-analysis which aimed to examine the association of overweight and obesity with premenopausal breast cancer, Amadou et al., [28] found that for BMI, each $5 \mathrm{~kg} / \mathrm{m}^{2}$ increase was inversely associated with the risk of premenopausal breast cancer $(\mathrm{RR}=0.95$; 95\% CI $=0.94-0.97)$.

Obesity is a predisposing factor for breast cancer especially in postmenopausal women, as circulating estrogen is primarily produced in fat tissue. Thus, having more fat tissue increases estrogen levels and the likelihood of having breast cancer [29]. Moreover, except of estrogens, body fatness directly affects circulating hormones such as insulin and insulin-growth factors, creating an environment that encourages carcinogenesis and discourages apoptosis. More specifically, insulin causes a reduction in sex-hormone binding globulin (SHBG) with a consequent elevation in bioavailable estradiol and stimulation of tumor cell proliferation. Insulin can also induce aromatase activity, thus producing an increase in estrogen biosynthesis that in adipose tissue or tumor cells could result in stimulation of breast cancer cell growth [30]. Furthermore, obesity seems to stimulate the body's inflammatory response firstly through adiponectin, an anti-inflammatory protein, and secondly through leptin which is mitogenic for breast cancer cells in vitro, and has biological activities that suggest its involvement in tumor cell angiogenesis and metastasis [30].

The strongest associations between individual cardiometabolic factors or components of metabolic syndrome with the risk of breast cancer have not been observed only for obesity but also for diabetes. In a recent meta-analysis which examined the potential of an increased risk of breast cancer in women with diabetes, the risk for the disease was increased by $27 \%$ (Summary Relative Risk (SRR) $=1.27 ; 95 \%$ $\mathrm{CI}=1.16-1.39$ ) for women with diabetes [31]. Moreover, a retrospective cohort study which aimed to compare breast cancer incidence between women with newly diagnosed diabetes $(n=73.796)$ to women without diabetes $(n=391.714)$, found a small but significant increase in incident breast cancer in the above postmenopausal population $(\mathrm{HR}=1.08$; $95 \%$ CI $=1.01-1.16 ; p=0.021$ ) [32]. Similarly, when Rosato et al., [33] analyzed the data of two Italian and Swiss casecontrol studies including again postmenopausal women, they found that the Odds Ratio (OR) of postmenopausal breast cancer was $1.33(95 \% \mathrm{CI}=1.09-1.62)$ for diabetes. At last, in the Nurses' Health Study which included 116.488 female nurses, those with type 2 diabetes had a modestly elevated incidence of breast cancer $(\mathrm{HR}=1.17 ; 95 \% \mathrm{CI}=1.01-1.35)$ compared with those without diabetes, independent of age, obesity, family history of breast cancer, history of benign breast disease, reproductive factors, physical activity and alcohol consumption [34].

Only a few studies provide evidence for a positive association of breast cancer with either hypertension or high cholesterol. Indeed, in a prospective study conducted in Finland, it was found that among women who were not using antihypertensive drugs $(\mathrm{AH})$ at baseline, the diastolic blood pressure (DBP) level was positively associated with the subsequent risk of breast cancer $(\mathrm{HR}=1.26 / 10 \mathrm{~mm} \mathrm{Hg} ; 95 \%$ $\mathrm{CI}=1.06-1.46$ ) [35]. However, in a case-control study conducted among women aged 50-75 years old, it was revealed that diuretic use was associated with elevated breast cancer risk $(\mathrm{OR}=1.79 ; 95 \% \mathrm{CI}=1.07-3.01)$ [36]. Concerning the association of low plasma HDL-cholesterol with risk of breast cancer, among 7.575 female members of the Atherosclerosis Risk in Communities Study (ARIC) cohort, a positive association for women who were premenopausal at baseline was found $(\mathrm{HR}=1.67 ; 95 \% \mathrm{CI}=1.06-2.63)$ [37]. Similarly, among 38.823 Norwegian women followed-up for a median of 17.2 years, low plasma HDL-cholesterol was associated with increased postmenopausal breast cancer risk [38].

\section{Limitations}

The major limitation of this study was the recall bias, as in all cases-controls studies. However, an effort was given to minimize this limitation by choosing newly diagnosed consecutive patients and collecting all necessary subjects in a small period of time. Moreover, people who collected the data were properly trained, limiting the bias between the investigators. The effect size measures used in case-control studies (i.e., the odds ratios) tend to overestimate the actual effect of the cause on effect usually observed in prospective studies; and thus, the findings should be interpreted with caution. 


\section{CONCLUSIONS}

The present study provides some evidence of a positive association of breast cancer with obesity, but not with other cardiometabolic risk factors; adding to the current scientific knowledge regarding cardiometabolic morbidities and breast cancer development.

\section{CONFLICT OF INTEREST}

The author(s) confirm that this article content has no conflicts of interest.

\section{ACKNOWLEDGEMENT}

The authors would like to thank all the participants of the study, without their contribution this project would never been done; also, would like to thank the Directors of the clinics of the involved hospitals: Gerasimos Aravantinos, Epaminondas Samantas, Evangelos Filopoulos, and the field investigators of the study: Aikaterini Manousou, Vassiliki Markasioti, Aikaterini Kakou, Fotini-Maria Mpalli, Nikolaos Soupos, Zoe Tsakalou, Maria Mitsou, Artemisia Tsakla, Iro Karapapa, Maria Somaraki, Athanasios Tektonidis, Athanasia Laina and Aggeliki Siolavou.

\section{REFERENCES}

[1] Grundy SM, Cleeman JI, Daniels SR, et al. Diagnosis and management of the metabolic syndrome: an American Heart Association/National Heart, Lung, and Blood Institute Scientific Statement. Circulation 2005; 112: 2735-52.

[2] Galassi A, Reynolds K, He J. Metabolic syndrome and risk of cardiovascular disease: a meta-analysis. Am J Med 2006;119: 8129.

[3] Gami AS, Witt BJ, Howard DE, et al. Metabolic syndrome and risk of incident cardiovascular events and death: a systematic review and meta-analysis of longitudinal studies. J Am Coll Cardiol 2007; 49: 403-14.

[4] Mottillo S, Filion KB, Genest J, et al. The metabolic syndrome and cardiovascular risk a systematic review and meta-analysis. J Am Coll Cardiol 2010; 56: 1113-32.

[5] Hubert HB, Feinleib M, McNamara PM, Castelli WP. Obesity as an independent risk factor for cardiovascular disease: a 26-year follow-up of participants in the Framingham Heart Study. Circulation 1983; 67: 968-77.

[6] Lavie CJ, Milani RV, Ventura HO. Obesity and cardiovascular disease: risk factor, paradox, and impact of weight loss. J Am Coll Cardiol 2009; 53: 1925-32.

[7] Marinou K, Tousoulis D, Antonopoulos AS, Stefanadi E, Stefanadis C. Obesity and cardiovascular disease: from pathophysiology to risk stratification. Int J Cardiol 2010;138: 3-8.

[8] Grundy SM, Benjamin IJ, Burke GL, et al. Diabetes and cardiovascular disease: a statement for healthcare professionals from the American Heart Association. Circulation 1999; 100: 1134-46.

[9] Howard BV, Magee MF. Diabetes and cardiovascular disease. Curr Atheroscler Rep 2000; 2: 476-81.

[10] Sowers JR, Epstein M, Frohlich ED. Diabetes, hypertension, and cardiovascular disease: an update. Hypertension 2001; 37: 1053-9.

[11] Esposito K, Chiodini P, Colao A, Lenzi A, Giugliano D. Metabolic syndrome and risk of cancer: a systematic review and metaanalysis. Diabetes Care 2012; 35: 2402-11.

[12] Kabat GC, Kim M, Chlebowski RT, et al. A longitudinal study of the metabolic syndrome and risk of postmenopausal breast cancer. Cancer Epidemiol Biomarkers Prev 2009;18: 2046-53.

[13] Bjorge T, Lukanova A, Jonsson $\mathrm{H}$, et al. Metabolic syndrome and breast cancer in the me-can (metabolic syndrome and cancer) project. Cancer Epidemiol Biomarkers Prev 2010; 19: 1737-45.
[14] Mourouti N, Papavagelis C, Psaltopoulou T, et al. Aims, design and methods of a case-control study for the assessment of the role of dietary habits, eating behaviors and environmental factors, on the development of breast cancer. Maturitas 2013; 74: 31-6.

[15] Panagiotakos DB, Pitsavos C, Stefanadis C. Dietary patterns: a Mediterranean diet score and its relation to clinical and biological markers of cardiovascular disease risk. Nutr Metab Cardiovasc Dis 2006; 16: 559-68.

[16] Panagiotakos D, Kalogeropoulos N, Pitsavos C, et al. Validation of the MedDietScore via the determination of plasma fatty acids. Int $\mathbf{J}$ Food Sci Nutr 2009; 60 Suppl 5: 168-80.

[17] Panagiotakos DB, Pitsavos C, Arvaniti F, Stefanadis C. Adherence to the Mediterranean food pattern predicts the prevalence of hypertension, hypercholesterolemia, diabetes and obesity, among healthy adults; the accuracy of the MedDietScore. Prev Med 2007; 44: 33540 .

[18] Craig CL, Marshall AL, Sjostrom M, et al. International physical activity questionnaire: 12 -country reliability and validity. Med Sci Sports Exerc 2003; 35: 1381-95

[19] Papathanasiou G, Georgoudis G, Papandreou M, et al. Reliability measures of the short International Physical Activity Questionnaire (IPAQ) in Greek young adults. Hellenic J Cardiol 2009; 50: 28394.

[20] Fountoulakis KN, lacovides A, Samolis S, et al. Reliability, validity and psychometric properties of the Greek translation of the Zung Depression Rating Scale. BMC Psychiatry 2001; 1: 6 .

[21] Zung WW. A self-rating depression scale. Arch Gen Psychiatry 1965; $12: 63-70$

[22] Fountoulakis KN, Papadopoulou M, Kleanthous S, et al. Reliability and psychometric properties of the Greek translation of the StateTrait Anxiety Inventory form Y: preliminary data. Ann Gen Psychiatry $2006 ; 5: 2$.

[23] Spielberger. CDGRLLRE:Manual for the State-Trait Anxiety Inventory (Self-Evaluation Questionnaire). Palo Alto, CA. Consulting Psychologists Press 1970.

[24] Cheraghi Z, Poorolajal J, Hashem T, Esmailnasab N, Irani DA. Effect of body mass index on breast cancer during premenopausal and postmenopausal periods: a meta-analysis. PLoS One 2012; 7 : e51446.

[25] Krebs EE, Taylor BC, Cauley JA, Stone KL, Bowman PJ, Ensrud KE. Measures of adiposity and risk of breast cancer in older postmenopausal women. J Am Geriatr Soc 2006; 54: 63-9.

[26] Lahmann PH, Hoffmann K, Allen N, et al. Body size and breast cancer risk: findings from the European Prospective Investigation into Cancer And Nutrition (EPIC). Int J Cancer 2004; 111: 762-71.

[27] Morimoto LM, White E, Chen Z, et al. Obesity, body size, and risk of postmenopausal breast cancer: the Women's Health Initiative (United States). Cancer Causes Control 2002; 13: 741-51.

[28] Amadou A, Ferrari P, Muwonge R, et al. Overweight, obesity and risk of premenopausal breast cancer according to ethnicity: a systematic review and dose-response meta-analysis. Obes Rev 2013; 14: 665-78.

[29] WCRF/AICR. Food, nutrition, physical activity, and the prevention of cancer: a global perspective. Washington, DC: AICR; 2007.

[30] Rose DP, Haffner SM, Baillargeon J. Adiposity, the metabolic syndrome, and breast cancer in African-American and white American women. Endocr Rev 2007; 28: 763-77.

[31] Boyle P, Boniol M, Koechlin A, et al. Diabetes and breast cancer risk: a meta-analysis. Br J Cancer 2012; 107: 1608-17.

[32] Lipscombe LL, Goodwin PJ, Zinman B, McLaughlin JR, Hux JE. Diabetes mellitus and breast cancer: a retrospective populationbased cohort study. Breast Cancer Res Treat 2006; 98: 349-56.

[33] Rosato V, Bosetti C, Talamini R, et al. Metabolic syndrome and the risk of breast cancer in postmenopausal women. Ann Oncol 2011; 22: 2687-92.

[34] Michels KB, Solomon CG, Hu FB, et al. Type 2 diabetes and subsequent incidence of breast cancer in the Nurses' Health Study. Diabetes Care 2003; 26: 1752-8.

[35] Lindgren A, Pukkala E, Tuomilehto J, Nissinen A. Incidence of breast cancer among postmenopausal, hypertensive women. Int $\mathrm{J}$ Cancer 2007; 121: 641-4. 
[36] Largent JA, McEligot AJ, Ziogas A, et al. Hypertension, diuretics and breast cancer risk. J Hum Hypertens 2006; 20: 727-32.

[37] Kucharska-Newton AM, Rosamond WD, Mink PJ, Alberg AJ, Shahar E, Folsom AR. HDL-cholesterol and incidence of breast cancer in the ARIC cohort study. Ann Epidemiol 2008; 18: 671-7.
[38] Furberg AS, Veierod MB, Wilsgaard T, Bernstein L, Thune I. Serum high-density lipoprotein cholesterol, metabolic profile, and breast cancer risk. J Natl Cancer Inst 2004; 96: 1152-60.

Received: October 09, 2013

Revised: October 11,2013

Accepted: October 11, 2013

(C) Mourouti et al.; Licensee Bentham Open.

This is an open access article licensed under the terms of the Creative Commons Attribution Non-Commercial License (http://creativecommons.org/licenses/by-nc/3.0/) which permits unrestricted, non-commercial use, distribution and reproduction in any medium, provided the work is properly cited. 